(REVIEW ARTICLE)

\title{
Fundamentals of extrachromosomal circular DNA in human cells - Genetic activities as regards cancer promotion alongside chromosomal DNA
}

\author{
Reinhard H. Dennin *
}

Formerly: Department of Infectious Diseases and Microbiology, University of Lübeck, UKSH, Campus Lübeck, Germany.

Publication history: Received on 17 November 2020; revised on 24 November 2020; accepted on 25 November 2020

Article DOI: https://doi.org/10.30574/wjarr.2020.8.2.0442

\begin{abstract}
In addition to chromosomal DNA (chr-DNA) and mitochondrial DNA, eukaryotic cells contain extrachromosomal DNA (ec-DNA). Analysed extrachromosomal circular DNA (ecc-DNA) accounts for up to 20\% of the total cellular DNA. EccDNAs contain coding and non-coding sequences originating from chr-DNA and mobile genetic elements (MGEs). MGEs include sequences such as transposons, which have the potential to move between different and the same DNA molecules, thereby, for example, causing rearrangements and inactivation of genes. Ecc DNAs have aroused interest in diseases such as malignancies and diagnostic procedures relating to this. A database to collect ecc-DNA has been established. Investigations are needed to find possible differences in sequences of chr-DNA after sequencing the whole cellular DNA (WCD), namely: chr-DNA plus ec-/ecc-DNA compared to chr-DNA, which is separated from ec-/ecc-DNA. Standards for sequencing protocols of WCD have to be developed that also reveal the sequences of ecc-DNA; this concerns "single-cell genomics" in particular.
\end{abstract}

Keywords: Extrachromosomal Circular DNA; Oncogenes; Cancer Promotion; Whole-Genome Sequencing (WGS); Database.

\section{Facts on ec-/ecc-DNA}

It is a long-known fact that eukaryotic cells, besides chr-DNA and mitochondrial DNA (mt-DNA), contain extrachromosomal DNA (ec-DNA) or extrachromosomal circular DNA (ecc-DNA) [1, 2, 3]. Microvesicles [4] and "extracellular vesicles" [5] containing ec-DNA constitute a particular situation. Mainly, research has focused on explicitly termed extrachromosomal circular DNA (ecc-DNA) in tissues of healthy human subjects [6] and plasma [7,8] of human origin. Moreover, research has focused on oncogenes [9] in cells containing ec-/ecc-DNA with sequences that code for them [10].

The size of ecc-DNA ranges from about $2 \mathrm{kbp}$ to $\geq 20 \mathrm{kbp}$ [11], $35 \pm 15 \mathrm{kbp}$ [12] up to 1-5 Mbp [13]. The sizes may depend on the source of cells: the smaller ones up to $100 \mathrm{kbp}$ in healthy cells $[12,14]$. Their share of the total cellular DNA varies from approximately $2 \%$ to $20 \%[12,15]$, depending on the subjects, tissues, and cells tested. The estimated number of ecc-DNA molecules ranges from at least 100 per cell and up to "thousands in cancers and cell lines" [16] depending on cells or tissues studied. Numerous reports indicate that tumor cells contain the highest share of ecc-DNA of the whole cellular DNA (WCD). Sparse information is available on the generation of ec-/ecc-DNA [17], in particular, concerning the transfer of chr-DNA derived sequences coding for oncogenes to ecc-DNAs [18].

Regarding the development of ec-/ecc-DNA from chr-DNA, there are various indications, such as "abnormal repair mechanisms", "genomic rearrangements ... involving circular DNA", and "self-joining" in the cited references. Models of formation are proposed, focusing on cancer pathogenesis [19] and following "environmental stress" [20]. For these

${ }^{*}$ Corresponding author: Reinhard H. Dennin

Formerly: Department of Infectious Diseases and Microbiology, University of Lübeck, UKSH, Campus Lübeck, Germany. 
processes, mobile DNA [21] or mobile genetic elements (MGE) may provide linking mechanisms between chr-DNA and the generation of ec-/ecc-DNAs. Because of their known and proven arsenal of corresponding elements (see below), they can contribute to transpose/move DNA sequences between different locations and join them to functional units.

\section{Facts about DNA sequences contained in ec-/ecc-DNA}

The different types of sequences in the ecc-DNA fraction indicate that they are derived from chr-DNA [3, 22]. This also applies to different types of sequences, which are referred to as MGE. MGE is a collective term for various genetic elements, such as Alu elements [23] and transposons. Estimates on their share of the WCD range from 50 to $80 \%$. MGE stands for DNA sequences with transposable potential known for their ability with the help of encoded enzymes upon stimulation to cut out sequence sections of chr-DNA and reintegrate them — randomly or purposefully? - at different locations of the chr-DNA (level 1) or into ec-/ecc-DNA (level 2) and vice versa ("jumping genes"); these kinds of rearrangements could possibly influence adjacent sequences containing regulatory or coding sections.

Reports support the interpretation that stressful situations [24] on cells can cause various genetic changes. The stress events focus on the effects such as environmental processes and carcinogens, leading to changes in the chr-DNA, such as genomic aberrations and instabilities. Here, a broad consensus concerns the activation of proto-oncogenes to form oncogenes $[9,25]$. The activation sequels and the metabolic consequences have drawn interest in the context with eccDNA.

Furthermore, sequences of a different kind exist with the potential to transpose or to regulate:

Sequences of human endogenous retroviruses (HERV) share about $8 \%$ of the human genome, and some of them are active [26]. Hints suggest "Tumor microvesicles contain retrotransposon elements ..." [4] and findings indicating that HERV-K elements may also be contributing to variations within the human genome [27].

Viral DNA sequences, with potential regulatory function due to the individual pattern of methylation, are present in the ecc-DNA fraction of peripheral blood mononuclear cells (PBMCs) of healthy subjects [12].

\section{Ecc-DNA associated with diseases focussing on cancer}

Apart from sequences of "chromosomal tandem repeats" [3], there are others with coding, promoting, and regulative potential assigned to ec-/ecc-DNA, which are known in the context of the development of tumors.

Thus far, the knowledge gained from research into ec-/ecc-DNA has deepened the understanding of how tumors (all?) may develop. The ecc-DNA in tumor cells contains sequences coding for oncogenes and is expressed in transcriptomes $[28,29,30,31]$. Furthermore, this concerns indications for "poorer outcomes" for various kinds of cancer patients [32], autoimmune disease [33], the involvement of ecc-DNA in "neurodegenerative" processes [34], and their "accumulation" in aging cells [13].

Although not systematically studied, it is believed that the composition and sequences of ecc-DNA change over time, causing extraordinary heterogeneities, and that it is also dependent on the tissue. Whether the high amount of ecc-DNA in cancer cells and "aging cells" is due to self-replication has not been sufficiently clarified yet [19]. Beyond the involvement of cancer-promoting properties, do ec-/ecc-DNAs have regular functions in the metabolism of cells controlled by a particular type of genetic communication? An indication for this situation may be the individual patterns of methylation of sequences of the 5'-NCR of the HCV contained in the ecc-DNA of healthy subjects' PMCs [12].

\section{Ecc-DNA and whole-genome sequencing (WGS)}

The ongoing processes for reorganizing the chr-DNA sequences are caused both by internal programming and probably the interactions between ec-/ecc-DNA segments and chr-DNA.

Based on the respective facts, the following situations should be considered paradigmatically. WCD subjected to sequencing protocols contains both chr-DNA and ec-/ecc-DNA; in particular, in cells of cancer tissue ec-/ecc-DNA containing coding sequences regarding the promotion of cancer are enriched. If ec-/ecc-DNA sequences after the WGS are not displayed, the results may be missing crucial information for medical purposes. Therefore, given such significant sequences, protocols for cell genomics have to be addressed. Does the exclusive use of WCD for WGS consider ecc-DNA reliably besides chr-DNA sequences? For example, regarding: i) the human genome itself, ii) for basic research, as well 
as for iii) targeted medical purposes, such as finding sequences, single ones or more being functional in clusters for certain genes that are or could be expected to be involved in diseases, diagnostics and therapy [35].

A special field of research is already based on single-cell genomics [36, 37]. However, caution is advised without considering: i) different amounts of ecc-DNA per cell, ii) their various genetic information, and iii) the use of single-cell diagnostics. The genetic heterogeneity of ecc-DNA in different cells, e.g., cancer cells, provides an unknown interpretation potential depending on the randomly caught cell(s). Single-cell genomics not considering the ecc-DNA are not appropriate to allocate the "clonal diversity to chromosomal and/or ecc-DNA [38].

\title{
5. Challenges
}

There are genetically active sections within the ec-/ecc-DNA fractions, such as those sequences promoting tumors. Consequently, the basic information obtained so far should enable the development of diagnostic tools, and concerning medical aspects such as therapy [39]. Depending on the subject/patient tested, if the results of WGS do not designate corresponding sequences of ecc-DNA, relevant information about the particular malignancy may be lost.

\section{Clarifications are needed}

Regarding the human genome, articles on "genes in pieces" based on the intron/exon postulates should also indicate whether the studies on human genomes have considered the possible influence of the known high shares of ec-/eccDNA [40] and their "reintegration" into the linear genome [29].

Given the ongoing alteration of genomes, the elaborated results after sequencing the WCD by WGS protocols seem to be just snapshots of just existing sequences of the chr-DNA. This situation applies to human "reference chromosomes" [41]. A protocol for the "...detection of ecc-DNA from NGS data" has been developed [42].

In particular, this holds for studies on cancer cells not considering ec-/ecc-DNA and the "single-cell" genomics [38]. A database for sequences in ecc-DNA has been established [43].

\section{Conclusion}

Ecc-DNAs can contain oncogene-coding sequences relevant for the promotion of cancer; ecc-DNA may be suitable to gain indications for the clinical-stage, therapeutic decisions, and drug resistance. Ecc-DNA should be considered in the respective diagnostics for patients with suspected cancer. The information obtained in this way reflects the most current and individual status.

Investigations are required to gain clarity as to whether sequences contained in the ec-/ecc-DNA fraction can affect the interpretation of sequences provided by sequencing the whole DNA (WDS) extracted from cells. Depending on the results, binding standards should be developed for protocols that determine sequences from human chr-DNA and ec/ecc-DNA. Only experimental controls with proven facts meet scientific standards.

\begin{abstract}
Abbreviations
Alu sequence elements, characterized by the restriction enzyme Alu I; ec-DNA, extrachromosomal DNA; ecc-DNA, extrachromosomal circular DNA; chr-DNA, chromosomal DNA; HCV, hepatitis C virus; HGP, human genome project; LINE: long interspersed element; MGE, mobile genetic elements; 5'-NCR, 5'-non-coding-region of the HCV-genome; PBMC, peripheral blood mononuclear cells; SINE: short interspersed element; WCD, whole cellular DNA; WGS, wholegenome sequencing
\end{abstract}

\section{Compliance with ethical standards:}

\section{Disclosure of conflict of interest}

No conflict of interest. 


\section{References}

[1] Sen S, Rani S, Freireich EJ, Hewitt R, Stass SA. Detection of Extrachromosomal Circular DNA Sequences From Tumor Cells by an Alkaline Lysis, Alu-polymerase Chain Reaction Technique. Mol Carcinog. 1992;5(2):107-110. doi:10.1002/mc.2940050205.

[2] Paulsen T, Kumar P, Koseoglu MM, Dutta A. New discoveries of extrachromosomal circles of DNA in normal and tumor cells. Trends Genet. 2018;34 (4):270-278. doi:10.1016/j.tig.2017.12.010.

[3] Cohen S, Segal D. Extrachromosomal circular DNA in eukaryotes: possible involvement in the plasticity of tandem repeats. Cytogenet Genome Res. 2009;124 (3-4):327-38. doi:10.1159/000218136.

[4] Balaj L, Lessard, R, Dai L, et al. Tumour microvesicles contain retrotransposon elements and amplified oncogene sequences. Nat Commun. 2011;2:180. doi:10.1038/ncomms180.

[5] Ferrari L, Cafora M, Rota F, et al. Extracellular Vesicles Released by Colorectal Cancer Cell Lines Modulate Innate Immune Response in Zebrafish Model: The Possible Role of Human Endogenous Retroviruses. Int. J. Mol. Sci. 2019;20:3669. doi:10.3390/ijms20153669.

[6] Møller, HD, Mohiyuddin M, Prada-Luengo I, et al. Circular DNA elements of chromosomal origin are common in healthy human somatic tissue. Nat Commun. 2018;9(1):1069. doi:10.1038/s41467-018-03369-8.

[7] Kumar P, Dillon LW, Shibata Y, et al. Normal and cancerous tissues release extrachromosomal circular DNA (eccDNA) into the circulation. Mol Cancer Res. 2017;15(9):1197-1205. doi:10.1158/1541-7786.MCR-17-0095.

[8] Sin STK, Jiang P, Deng J, et al. Identification and characterization of extrachromosomal circular DNA in maternal plasma. PNAS USA. 2020; 117(3):1658-1665. doi:10.1073/pnas.1914949117.

[9] Cooper GM. The Cell: A Molecular Approach. 2nd edition. Sunderland (MA): Sinauer Associates; 2000. Oncgenes. https://www.ncbi.nlm.nih.gov/books/NBK9840/

[10] $\mathrm{Gu} \mathrm{X,} \mathrm{Yu} \mathrm{J,} \mathrm{Chai} \mathrm{P}$, et al. Novel insights into extrachromosomal DNA: redefining the onco-drivers of tumor progression. J Exp Clin Cancer Res. 2020; 39:215.https://doi.org/10.1186/s13046-020-01726-4.

[11] Cohen S, Agmon N, Sobol O, Segal D. Extrachromosomal circles of satellite repeats and 5S ribosomal DNA in human cells. Mob DNA. 2010;1:11. doi:10.1186/1759-8753-1-11.

[12] Dennin RH, Wo J. DNA sequences homologous to hepatitis C virus (HCV) in the extrachromosomal circular DNA in peripheral blood mononuclear cells of HCV-negative subjects. J Zhejiang Univ Sci B. 2019;20(8):637-646. doi:10.1631/jzus.B1800453.

[13] Hull RM, Houseley J. The Adaptive Potential of Circular DNA Accumulation in Ageing Cells. Curr Genet. 2020;66(5). doi:10.1007/s00294-020-01069-9. Online ahead of print.

[14] Bailey C, Shoura MJ, Mischel PS, Swanton C. Extrachromosomal DNA - relieving heredity constraints, accelerating tumour evolution. Ann Oncol. 2020; S0923-7534 (20)36392-4. doi:10.1016/j.annonc.2020.03.303.

[15] Schmidt H, Taubert H, Lange H, et. al. Small polydispersed circular DNA contains strains of mobile genetic elements and occurs more frequently in permanent cell lines of malignant tumors than in normal lymphocytes. Oncol Rep. 2009; 22(2):393-400. doi:10.3892/or 00000450.

[16] Kumar P, Kiran Sh, Saha Sh, Su Z, Paulsen P, Chatrath A, et al. ATAC-seq identifies thousands of extrachromosomal circular DNA in cancers and cell lines. Sci Adv. 2020 May;6(20):eaba2489. doi:10.1126/sciadv.aba2489.

[17] van Loon N, Miller D, Murnane JP. Formation of extrachromosomal circular DNA in HeLa cells by nonhomologous recombination. Nuc Acids Res. 1994;22 (13):2447-2452. doi:10.1093/nar/22.13.2447.

[18] Verhaak RGW, Bafna V, Mischel PS. Extrachromosomal oncogene amplification in tumour pathogenesis and evolution. Nat Rev Cancer. 2019;19:283-288. doi:10.1038/s41568-019-0128-6.

[19] Zhenyu L, Wang J, Ye L, Li T, Yu X, Liu L, et al. Classification of extrachromosomal circular DNA with a focus on the role of extrachromosomal DNA (ecDNA) in tumor heterogeneity and progression. Biochimica et Biophysica Acta (BBA) - Reviews on Cancer. 2020 Aug;1874(1):18839.

[20] Yan Y, et al. Current understanding of extrachromosomal circular DNA in cancer pathogenesis and therapeutic resistance. J Hematology \& Oncology. 2020;13:124. doi.org/10.1186/s13045-020-00960-9. 
[21] Kazazian HH, Moran JV. Mobile DNA in Health and Disease. N Engl J Med. 2017 July 27;377(4):361-370. doi:10.1056/NEJMra1510092.

[22] Shoura MJ, Gabdank I, Hansen L, et al. Intricate and Cell Type-Specific Populations of Endogenous Circular DNA (eccDNA) in Caenorhabditis elegans and Homo sapiens. Genes, Genomes, Genetics. 2017;7:3295-3303. doi: https://doi.org/10.1534/g3.117.300141.

[23] Ade C, Roy-Engel AM, Deininger PL. Alu elements: An intrinsic source of human genome instability. Curr Opin Virol. 2013 December;3(6):639-645. doi:10.1016/j.coviro.2013.09.002.

[24] Toyokuni A, Critical Review - Molecular Mechanisms of Oxidative Stress-induced Carcinogenesis: From Epidemiology to Oxygenomics. IUBMB Life. 2008; 60(7):441-447. doi:10.1002/iub.61.

[25] Chial, H. (2008) Proto-oncogenes to oncogenes to cancer. Nature Education. 1(1):33.

[26] Grandi N, Tramontano E. Human endogenous retroviruses are ancient acquired elements still shaping innate immune responses. Front Immunol. 2018; 9: 2039. doi: 10.3389/fimmu.2018.02039.

[27] Shin W, Lee J, Son SY, et al. Human-specific HERV-K insertion causes genomic variations in the human genome. PLoS ONE. 2013;8(4):e60605. doi:10.1371/journal.pone.0060605.

[28] Wu S, Turner KM, Nguyen N, et al. Circular ecDNA promotes accessible chromatin and high oncogene expression. Nature. 2019;575:699-703. https://doi.org/10.1038/s41586-019-1763-5.

[29] Koche RP, Rodriguez-Fos E, Helmsauer K, et. al. Extrachromosomal circular DNA drives oncogenic genome remodelling in neuroblastoma. Nat Genet. 2020; 52 (1): 29-34. doi: 10.1038/s41588-019-0547-z.

[30] Morton, A. R., Nergiz Dogan-Artun, Zachary J. Faber, Mathieu Lupien. Jeremy N. Rich, Peter C. Scacheri. Functional Enhancers Shape Extrachromosomal Oncogene Amplifications. 2019. Cell 179, (6) P1330-1341. https://doi.org/10.1016/j.cell.2019.10.039.

[31] Tandon I, Pal R, Pal JK, Sharma NK. Extrachromosomal circular DNAs: an extra piece of evidence to depict tumor heterogeneity. Future Sci OA. 2019;5(6):FSO390. doi:10.2144/fsoa-2019-0024.

[32] Kim, H, Nguyen, N, Turner, K, et al. Extrachromosomal DNA is associated with oncogene amplification and poor outcome across multiple cancers. Nat Genet 2020;52:891-897. doi.org/10.1038/s41588-020-0678-2.

[33] Thomas Ch A, Tejwani L, Trujillo CA, et al. Modeling of TREX1-Dependent Autoimmune Disease using Human Stem Cells Highlights L1 Accumulation as a Source of Neuroinflammation. Cell Stem Cell. 2017;21:319-331. doi.org/10.1016/j.stem.2017.07.009.

[34] Ain Q, Schmeer C, Wengerodt D, Witte OW, Kretz A. Extrachromosomal Circular DNA: Current Knowledge and Implications for CNS Aging and Neurodegeneration. Int J Mol Sci. 2020;21(7):2477. doi:10.3390/ijms21072477.

[35] Christiansen JH, Nguyen N, Verhaak R, et al. Extrachromosomal DNA (ecDNA) carrying amplified oncogenes as a biomarker for insensitivity to pembrolizumab treatment in gastric cancer patients. J Clin Oncol 2020:38,(15) suppl. 3123; doi: 10.1200/jco.2020.38.15_suppl.3123.

[36] Han X, Zhou Z, Fei L, et al. Construction of a human cell landscape at single-cell level. Nature. 2020. doi.org/10.1038/s41586-020-2157-4.

[37] Wang S, Zheng Y, Li J, et al. Single-Cell Transcriptomic Atlas of Primate Ovarian Aging. Cell. 2020;180(3):585600.e19. doi.org/10.1016/j.cell.2020.01.009.

[38] Morita, K, Wang, F, Jahn, K, et al. Clonal evolution of acute myeloid leukemia revealed by high-throughput singlecell genomics. Nat Commun 2020;11:5327. doi.org/10.1038/s41467-020-19119-8.

[39] Yan, Y., Guo, G., Huang, J. et al. Current understanding of extrachromosomal circular DNA in cancer pathogenesis and therapeutic resistance. J Hematol Oncol 2020;13,124. doi.org/10.1186/s13045-020-00960-9.

[40] Smithers B, Oates M, Gough J. 'Why genes in pieces?'--revisited. Nucleic Acids Res. 2019;47(10):4970-4973. doi:10.1093/nar/gkz284.

[41] Ballouz S, Dobin A, Gillis JA. Is it time to change the reference genome? Genome Biol. 2019;20:159. doi.org/10.1186/s13059-019-1774-4.

[42] Mann L, Seibt K, Weber B, Schmidt Th. ECCsplorer: detection of extrachromosomal circular DNA (eccDNA) from NGS data. Biopolis Dresden PhD Symposium 2020, CRTD, Dresden, Germany, February 2020. https://doi.org/10.5281/zenodo.4017245. 
World Journal of Advanced Research and Reviews, 2020, 08(02), 279-284

[43] Sun H. Shenzhen Baoan Women's and Children'sHealth Hospital, Jinan University, 518102, Shenzhen, China. The extrachromosomal circular DNA database. 2020. www.eccdnadb.net 